\title{
Kinematic variables of gait and quality of life in Parkinsonians after different treadmill trainings: a randomized control trial
}

\author{
Maira Peloggia Cursino ${ }^{1 *}$, Doralice Fernanda Raquel ${ }^{2}$, Camilla Zamfolini Hallal ${ }^{3}$, Flávia \\ Roberta Faganello Navega ${ }^{4}$
}

\begin{abstract}
The aim of this study was to analyse and compare the effects of gait training on a treadmill associated with partial body weight support (PBWS) or auditory stimulus (AS) and the kinematic variables of gait and quality of life (QOL) in patients with Parkinson's disease (PD). Twenty-one volunteers with PD participated and were divided into: group with PBWS (GPBWS) with AS (GAS), and control group (CG). Soil step length (SL), SL variability (SLV), step width (SW), SW variability (SWV), gait speed (GS), and QOL were evaluated by the Parkinson's Disease Questionnaire-39 (PDQ-39). The training was performed for six weeks, with three weekly sessions of 30 minutes. The results showed homogeneity of groups $(p>0.05)$. There was an increase of SW in the GPBWS $(p=0.006)$ and GS in the GAS $(p=0.048)$ and decreased PDQ39 in the CG $(p=0.005)$ and GAS $(p=0.006)$. For groups, regardless of evaluation, there were differences in SWV between the GPBWS and GAS $(p=0.030)$; for evaluations, independently of groups, there were differences in GS $(p=0.048)$ and PDQ-39 ( $p=0.002)$. It was concluded that, among the studied conditions, there was a significant improvement for the groups: AS in GS and QOL, CG in QOL and GPBWS did not improve. Thus, considering the clinical effect, treadmill training, independently of the stimuli, is clinically advantageous for gait improvements and QOL.

Keywords: Parkinson Disease, Quality of Life, Walking, Biomechanical Phenomena, Randomized Control Trial
\end{abstract}

\section{INTRODUCTION}

The Parkinson's disease (PD) is the second most common neurodegenerative disorder and is the one that causes most movement disorders worldwide (Rodriguez, Roemmich, Cam, Fregly, \& Hass, 2013). The main symptoms of PD are tremor at rest, muscle rigidity, bradykinesia and postural instability. It is a progressive and chronic disease, and the development of signs and symptoms contributes to physical and mental impairment, influencing day-to-day life and worsening quality of life (QOL) (Scalzo, Flores, Marques, Robini, \& Teixeira, 2012).

Exercise can reduce motor and non-motor disturbances of PD (Abbruzzese, Marchese, Avanzino, \& Pelosin, 2016), such as changes in gait and QOL, since gait performance is a major determinant of independence and QOL (Del
Olmo \& Cudeiro, 2005). In addition, gait disorders are among the leading and most disabling symptoms of $\mathrm{PD}$, which are associated with changes in balance, which affects the implementation of independent and secure gait (Bello et al., 2013). The debilitating effects on gait are decreased step length (SL) and step speed, increased gait variability, increased duration of the double support phase, reduced range of motion of the triple flexion, increased cadence, and gait festination (Matsumoto, Magalhães, Antunes, \& Torriani-Pasin, 2014; Rodriguez et al., 2013).

When changes in walking in PD patients are observed, a treadmill workout with constant speed encourages an unconscious automatism and allows for motor and memory learning through repetition of a specific task more strongly

\footnotetext{
Manuscript received at February $2^{\text {nd }} 2017$; Accepted at October $22^{\text {nd }} 2017$

${ }^{1}$ Universidade Estadual Paulista Julio de Mesquita Filho, Marília, Brazil

${ }^{2}$ Universidade Estadual Paulista Julio de Mesquita Filho, Marília, Brazil

${ }^{3}$ Universidade Federal de Uberlândia, Uberlândia, Brazil

${ }^{4}$ Universidade Estadual Paulista Julio de Mesquita Filho, Marília, Brazil

* Corresponding author: Av. Hygino Muzzi Filho, 737, Bairro: Mirante, 17.525-900 - Marília, SP, Brazil E-mail: maira_cursino@hotmail.com
} 
than when walking outside (Harris-Love, Macko, Whitall, \& Forrester, 2004; Herman, Giladi, Gruendlinger, \& Hausdorff, 2007). Thus, the neuroplasticity of the brain, including the basal ganglia, may be affected by PD (Nadeau, Pourcher, \& Corbeil, 2014), making the treadmill an excellent option for rehabilitation (HarrisLove et al., 2004).

When a treadmill workout is associated with partial body weight support (PBWS), it can improve mobility and balance because it facilitates the movement of the lower limbs and is quite safe from falls, making it favourable for improving locomotion in people with PD (Ganesan, Sathyaprabha, Gupta, \& Pal, 2014). This method has been proven effective in functional gait in other populations with neurological disorders, such as individuals with hemiparesis (Harris-Love et al., 2004), and generates longer effects for cadence and gait speed (GS) than traditional physical therapy (Miyai et al., 2002).

External sensory stimuli have been successfully used in patients with central nervous system afflictions, such as PD, to improve the gait pattern (Suteerawattananon, Morris, Etnyre, Jankovic, \& Protas, 2004). The use of auditory stimuli (AS) by techniques such as music and metronomes is a good strategy for patients with $\mathrm{PD}$, because it associates a motor task with cognitive attention (Earhart \& Williams, 2012). The SL and cadence during the gait are also significantly higher with the use of an auditory sensory trail in PD patients, and the time needed for the muscles to respond to the motor command is reduced (Del Olmo \& Cudeiro, 2005). Thus, the AS can assist in synchronizing the movement and make the recruitment pattern of motor units more consistent, which can be beneficial if it is associated with the gait (Miller, Thaut, Mcintosh, \& Rice, 1996).

Gait training on a treadmill is available in most physical therapy clinics and is advantageous because it can result in improvements in GS, SL, and cadence in people with PD (Earhart \& Williams, 2012). Although many gait training methods for PD patients have been proposed in the literature, there are no studies that show through biomechanics, kinematics variables or assessment of QOL that the PBWS or AS associated with gait training on a treadmill improves the standard of locomotion and Parkinsonian QOL. Thus, the prevention and treatment of disorders in gait in PD patients can be optimized to detect effective interventions and improve gait patterns, guiding physiotherapeutic treatments.

The objective of this study was to analyse and compare the effects of gait training on a treadmill in PD patients with PBWS and gait training with AS on the kinematic variables of gait SL, SL variability (SLV), step width (SW), SW variability (SWV), and usual GS on QOL.

In view of the findings in the literature presented in this document, we hypothesized that treadmill training associated with AS or BWPS promotes significant changes in biomechanical and kinematic variables of gait, such as increased GS, the SL and decreased SW, the VSL, SWV, and QOL test when compared to treadmill training without stimuli.

\section{METHODS}

This study is a randomized controlled clinical trial. A block randomization was used. Participants were recruited from March 2014 to August 2015 through advertisements on television, leafleting at bus terminals, medical offices, hospitals, basic health units, as well as patient charts in referral hospitals in the region of Marília/SP and recommendations of physiotherapy services of the city.

\section{Participants}

The convenience sample was based on a sample size calculation. The study included 21 participants (12 males and 9 females) with an idiopathic PD diagnosis made by a doctor, classified in stages I to III of the Hoehn and Yahr scale (Hoehn \& Yahr, 1967), in which it is possible to perform independent walking.

Eligibility criteria included the ability to walk without the use aid devices; no participation in a pharmacological adaptation phase; carry out the evaluations and interventions in phase "on" drugs for PD; and no pain, fracture, serious injury to soft tissue in the six months before the study, severe osteoporosis, history of cognitive and 
other neurological impairments, or uncontrolled cardiovascular or respiratory changes or other chronic uncontrolled conditions that may interfere with the safety and performance of the training protocol and testing. For the assessment of cognitive function, we used the Mini-Mental State Examination (MMSE), and cut-off points were used for the participant's schooling (Brucki,
Nitrini, Caramelli, Bertolucci, \& Okamoto, 2003).

The 21 study participants were randomly divided into three groups: 7 in the group with partial body weight support (GPBWS), 7 in the group with auditory stimulus (GAS), and 7 in the control group (CG) (Figure 1).

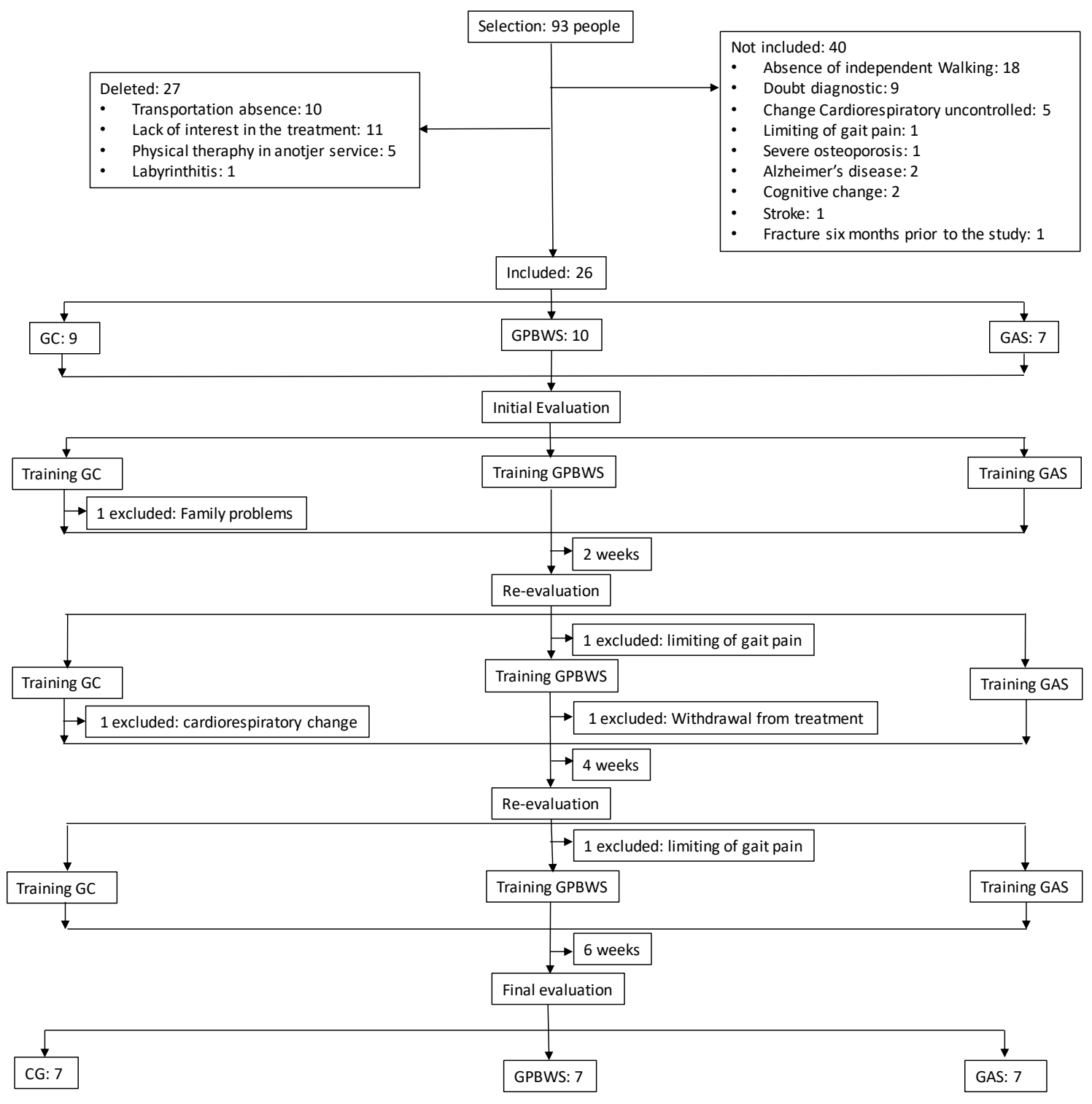

Figure 1. Flowchart distribution of participants

This study was approved by the Ethics Committee of the Faculty of Philosophy and Sciences of UNESP-Marilia (no. 0967/2014) and the Platform Brazil (no. 1054261), and it was registered in the Brazilian Registry of Clinical Trials (RBR-6bgqk4). After having read and agreed to the free and informed consent form, the participants signed the form according to
Resolution 466/2012 and its complement of the National Health Council.

\section{Measures}

To collect kinematic gait data on SL, SLV, SW, and SWV, we used two Sony ${ }^{\circledR}$ Handycam DCRSR68E cameras with a $60 \mathrm{~Hz}$ sampling frequency (Japan), one in the frontal plane, which was used 
for the analysis of the posterior view, that is, the SW, and another in the sagittal plane used for the analysis of the lateral view, that is, the SL, as shown in the figure 2 and an oval circuit $(4.8 \mathrm{~m} \mathrm{x}$ $3.2 \mathrm{~m}$ ) that enables continuous gait and photoreflective markers for the identification of the trochanter, line lateral side of the knee joint, lateral malleolus, and calcaneus on the right side of the body, and the medial malleolus and calcaneus on the left side of the body.

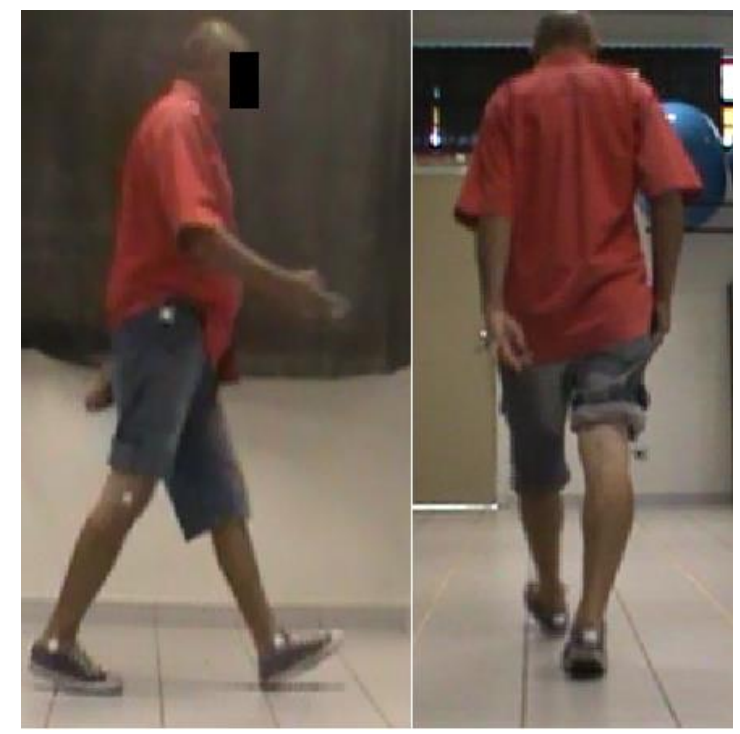

Figure 2. Photoreflexive markers on the anatomical points in the right side: trochanter, joint line of the knee, lateral malleolus and calcaneus; In the left side: medial malleolus and calcaneus, used for analysis of the length and step width.

Camera calibration was performed before the start of each film using a known measure square $(1 \mathrm{~m} \times 1 \mathrm{~m})$, as shown in the figure 3 . The calibration square was placed standing with one edge propping on the ground and turned to both cameras, focused by 10s, thus allowing all edges to be filmed. This calibration is used as the basis for the analysis of kinematic data of gait in the floor. After calibration, the camera was kept in the same position so as not to change the collected data, which were subsequently analysed in the Peak Motus Motion Measurement System, version 9.0 (USA). This program records the position and movement of the body segments, according to the marking of joint points of interest and to control the acquisition reading, scanning, processing and storage of kinematic parameters for synchronization and subsequent data analysis.
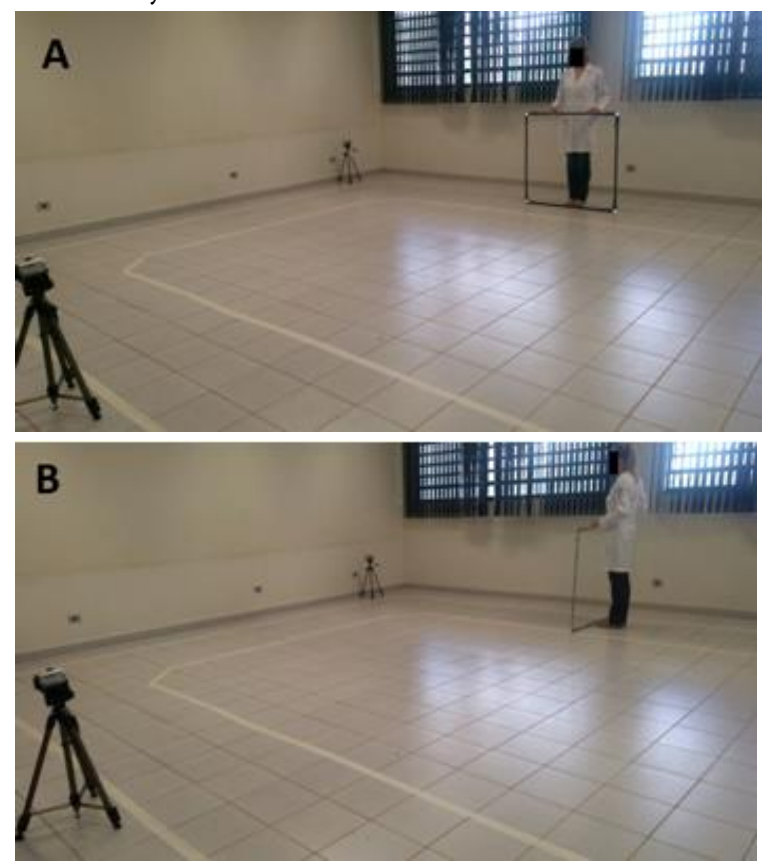

Figure 3. Calibration of cameras before the participation of the volunteer. (A) Side view camera calibration. (B) Posterior view camera calibration

The evaluation of the usual GS was made using the $10 \mathrm{~m}$ walk test $(10 \mathrm{mWT})$. For this evaluation, the participants walked at their normal speed a distance of $12.4 \mathrm{~m}$, in which the first and final $1.2 \mathrm{~m}$ were disregarded to eliminate acceleration and deceleration component. Thus, the participants walked straight, and the timer was activated at the $1.2 \mathrm{~m}$ mark and disabled after the participant walked $10 \mathrm{~m}$, reaching the mark of the final $1.2 \mathrm{~m}$. This test was performed three times and then calculated by the arithmetic mean of the values obtained in the three attempts (Novaes, Miranda, \& Dourado, 2011).

We used the Parkinson's Disease Questionnaire - 39 (PDQ-39) for the assessment of QOL. The Brazilian version of the PDQ-39 is considered a reliable and valid measure for use in patients with PD (Carod-Artal, Martinez-Martin \& Vargas, 2007). The PDQ-39 is a specific questionnaire for $\mathrm{PD}$ that assesses eight key areas of health and daily activities (mobility - 10 items, activities of daily living - 6 items, emotional wellbeing - 6 items, stigma - 4 items, social support 3 items, cognition - 4 items, communication - 3 items, and bodily discomfort -3 items). The score 
for each item ranges from 0 to 4 points, and the lower score reflects higher QOL.

All intervention procedures were performed on an electric treadmill (Fitness Evolution EVO 4000, Brazil) with the use of a safety belt attached to a steel cable connected to a metal support structure to prevent falls during training. The GPBWS used the load cell (EMG System Brazil®) for PBWS setting, and the GAS used the Metronome (Metronome Plus, Second Edition, version 2.0.0.5, $\mathrm{M} \& \mathrm{M}$ Systems, Germany) connected to a sound box (Master ${ }^{\circledR}$ Audio and Amplifier, Brazil) for auditive signals.

\section{Procedures}

On the first day, the participants were instructed in detail about the objectives and procedures of the study, and after that they performed the PDQ-39 questionnaire and, $10 \mathrm{mWT}$, which was used as the basis for treadmill training and the biomechanical evaluation of gait on the floor.

For evaluation of gait, the participants walked at their preferred speed without interruptions in the collection area for five consecutive times to familiarize themselves with the circuit and five times to collect 10 steps in the calibrated area for gait evaluation. Studies show that changes in gait parameters are more easily identified in continuous situations than in a restricted space, because the latter, with continual interruptions, causes changes in the temporal locomotor rhythm and may result in adverse effects on pattern analysis of gait (Paterson, Lythgo, \& Hill, 2009).

Before the intervention, the participants selected their preferred GS on the treadmill. Participants were initially subjected to a speed $50 \%$ lower than that obtained in the $10 \mathrm{mWT}$. After the participants became familiar with the operation of the treadmill, the speed was increased until the participant walked faster than usual, and this value was registered. Subsequently, the speed was reduced until it was slower than the participant's day-to-day GS, and this value was registered. After performing this procedure three times, speeds were averaged (Dingwell, \& Marin, 2006), and the familiarization and training speeds were set.
Familiarity was accomplished according to the protocol proposed by Bello, Sanchez, and Fernandez-del-Olmo (2008). To avoid excessive fatigue, the participants were encouraged to take breaks when needed throughout the study procedures (Mirelman et al., 2013).

Training sessions for the GPBWS, GAS, and CG were individual and were held three times a week for six consecutive weeks. Each training session was 30 minutes long. The blood pressure of each participant was measured before, during, and after each workout. Every two weeks, the participants' body mass was measured, as well as the evaluation of the preferred speed on the ground and on the treadmill, for adjustments in training speed. The familiarization was performed with the speed obtained to continue the training days.

The GPBWS performed gait training on a treadmill at $20 \%$ of adjusted body weight support by the load cell, preferably at the predetermined velocity for each participant. The percentage of PBWS was selected based on previous studies (Ganesan et al, 2014; Miyai et al., 2002).

GAS performed gait training on a treadmill at the predetermined preferred speed associated with AS coming from an adjusted metronome at a rate $20 \%$ lower than the average frequency step gait on a treadmill. This stimulation was used to induce an increase in SL by decreasing gait cadence at a constant speed. The CG performed the entire gait training on a treadmill at a predetermined preferred speed not associated with any other type of intervention. The treadmill intervention for the CG was selected to isolate the effects of training with PBWS and AS, since the literature indicates improvements in PD patients' gait pattern after intervention with gait on a treadmill (Bello et al., 2013).

The protocol assessments were performed before and after six weeks of intervention, and each participant was evaluated at approximately the same time of day to avoid variability of performance due to any circadian rhythms or medication intake cycle.

\section{Statistical Analysis}

For the kinematic gait analysis, the first 10 steps inside of the calibrated area of the oval 
circuit were measured, both for the SL, using the lateral camera, and SW, using the posterior camera, and the arithmetic mean of these 10 steps was performed. The normalization of lower limb length was also performed according to the formula:

$$
\frac{\text { average SL }}{\text { right lower limb size }} \times 100
$$

For the analysis of GS, 10Mwt was used, and to evaluate the QOL the PDQ-39 questionnaire score was used.

Statistical analysis was performed with ANOVA Two Way Repeated Measures with Post Hoc test of Bonferroni through PASW statistics $18.0{ }^{\circledR}$ software. A significance level of $\mathrm{p}<0.05$ was adopted in all statistical tests. The effect size measures and magnitude of the effect (d) comparing the initial and final moments were calculated for all variables according to the equation:

$$
d=\frac{\operatorname{Mean}^{F}-\operatorname{Mean}^{I}}{S}
$$

where Mean ${ }^{\mathrm{F}}$ is the mean final value, Mean ${ }^{\mathrm{I}}$ is the mean initial value, and $S$ is the pooled standard deviation. The d-values between 0.20 and 0.49 were considered a small effect, d-values between 0.50 and 0.79 a medium effect, and dvalues $\geq 0.80$ a large effect (Cohen, 1992). The $d$ tries to replace the concept of statistical significance with more useful notions of significance in the specific practice (Conboy, 2003).

Table 1.

Values expressed as mean (standard deviation) age, body mass, height, BMI, diagnostic time, Hoehn and Yahr, Mini-Mental State Examination, and falls to characterize the sample.

\begin{tabular}{lcccccccc}
\hline Group & Age (Years) & $\begin{array}{c}\text { Body mass } \\
(\mathrm{Kg})\end{array}$ & Height $(\mathrm{m})$ & $\begin{array}{c}\text { BIM } \\
\left(\mathrm{Kg} / \mathrm{m}^{2}\right)\end{array}$ & $\begin{array}{c}\text { Diagnosis } \\
\text { time (years) }\end{array}$ & $\begin{array}{c}\text { Hoehn and } \\
\text { Yahr }\end{array}$ & MMSE & $\begin{array}{c}\text { Falls } \\
(\text { last year })\end{array}$ \\
\hline CG & $72(10.52)$ & $68.50(12.14)$ & $1.63(0.07)$ & $25.90(4.19)$ & $6.29(3.35)$ & $2.29(0.95)$ & $25.57(2.76)$ & $2.14(2.27)$ \\
GBPWS & $63.29(11.06)$ & $75.87(11.50)$ & $1.67(0.12)$ & $27.07(1.66)$ & $2.86(1.57)$ & $2(0.82)$ & $27.29(1.89)$ & $2.29(4.35)$ \\
GAS & $70(7.23)$ & $71.29(10.51)$ & $1.62(0.09)$ & $27.28(3.69)$ & $4.14(2.61)$ & $1.71(0.95)$ & $27.86(2.04)$ & $0.29(0.49)$ \\
\hline
\end{tabular}

CG = control group $(n=7)$; GPBWS = partial body weight support group $(n=7)$; GAS = auditory stimulus group $(n=7)$; MMSE $=$ Mini-Mental State Examination. $\mathrm{p}>0.05$

Table 2.

Results of the initial and final evaluations of the kinematic variables of gait: step length, step length variability, step width, step

\begin{tabular}{|c|c|c|c|c|c|c|c|c|c|}
\hline & \multicolumn{3}{|c|}{$\mathrm{CG}$} & \multicolumn{3}{|c|}{ GPBWS } & \multicolumn{3}{|c|}{ GAS } \\
\hline & Initial & Final & $\mathrm{d}$ & Initial & Final & $\mathrm{d}$ & Initial & Final & $\mathrm{d}$ \\
\hline SL & $59.20(5.24)$ & $64.68(5.88)$ & 0.98 & $65.83(2.50)$ & $65.57(2.85)$ & 0.10 & $66.69(2.73)$ & $68.67(2.91)$ & 0.70 \\
\hline SLV & $5.55(0.32)$ & $5.81(0.74)$ & 0.40 & $5.98(0.47)$ & $5.28(0.63)$ & 1.23 & $5.61(0.50)$ & $4.86(0.55)$ & 1.42 \\
\hline SW & $0.11(0.01)$ & $0.09(0.01)$ & 2.00 & $0.06(0.01)$ & $0.08(0.01)^{*}$ & 2.00 & $0.08(0.01)$ & $0.07(0.01)$ & 1.00 \\
\hline SWV & $0.05(0.01)$ & $0.05(0.00)$ & 0.00 & $0.05(0.01)$ & $0.06(0.01)$ & 1.00 & $0.04(0.00)$ & $0.04(0.00)$ & 0.00 \\
\hline GS & $0.99(0.11)$ & $1.09(0.09)$ & 0.99 & $1.20(0.06)$ & $1.24(0.05)$ & 0.72 & $1.16(0.08)$ & $1.24(0.06)^{*}$ & 1.11 \\
\hline PDQ-39 & $103.57(6.86)$ & $87.00(8.20)^{*}$ & 2.17 & $79.71(7.76)$ & $71.29(7.62)$ & 1.09 & $90.29(6.70)$ & $72.29(5.65) *$ & 2.88 \\
\hline
\end{tabular}
width variability, gait speed, and quality of life presented as mean (standard deviation) and magnitude of the effect (d).

$\mathrm{CG}=$ control group $(\mathrm{n}=7)$; GPBWS = partial body weight support group $(\mathrm{n}=7)$; GAS = auditory stimulus group ( $\mathrm{n}=7$ ); $=$ magnitude of the effect (small effect $\mathrm{d}$ between 0.20 and 0.49 , medium effect $\mathrm{d}$ between 0.50 and 0.79 , and large effect $\mathrm{d} \geq$ 0.80); SL = step length (cm); SLV = step length variability; SW = step width (cm); SWV = step width variability; GS = gait speed (m/s); PDQ-39 = Parkinson's Disease Questionnaire - 39; * Evaluation different than re-evaluation intragroup; p<0.05.

Table 3.

Mean (standard deviation) of step length, step length variability, step width, step width variability, gait speed, and Parkinson's Disease Questionnaire - 39 in the analysis groups and evaluation.

\begin{tabular}{lccccc}
\hline & \multicolumn{3}{c}{ Group } & \multicolumn{2}{c}{ Assessment } \\
\cline { 2 - 6 } SL & CG & GPBWS & GAS & Initial & Final \\
nyL & $61.94(5.27)$ & $65.70(2.55)$ & $67.68(2.73)$ & $63.90(2.52)$ & $66.31(3.00)$ \\
SW & $5.68(0.38)$ & $5.63(0.41)$ & $5.24(0.41)$ & $5.71(0.21)$ & $5.32(0.30)$ \\
SWV & $0.10(0.01)$ & $0.07(0.01)$ & $0.07(0.01)$ & $0.08(0.00)$ & $0.08(0.01)$ \\
GS & $0.05(0.00)$ & $0.05(0.01)^{*}$ & $0.04(0.00)$ & $0.04(0.00)$ & $0.05(0.00)$ \\
PDQ-39 & $1.04(0.10)$ & $1.22(0.06)$ & $1.20(0.07)$ & $1.12(0.05)$ & $1.19(0.04) * *$ \\
\hline
\end{tabular}

CG = control group $(n=7)$; GPBWS = partial body weight support group $(\mathrm{n}=7)$; GAS = auditory stimulus group ( $\mathrm{n}=7$ ); $\mathrm{SL}=$ step length $(\mathrm{cm})$; SLV = step length variability; SW = step width $(\mathrm{cm}) ; \mathrm{SWV}=$ step width variability; GS = gait speed $(\mathrm{m} / \mathrm{s})$; PDQ-39 = Parkinson Disease Questionnaire - 39; *GPBWS is different from GAS; ${ }^{*}$ Evaluation different than reevaluation independent of the group. 


\section{RESULTS}

Table 1 shows the characterization of the sample. Statistical analysis showed no difference between groups for the characteristics studied ( $\mathrm{p}>0.05)$.

Statistical analysis indicated that there was a main effect for the groups in the SWV $\left(\mathrm{F}_{(2,12)}=\right.$ 5.076; $\mathrm{p}=0.025)$ and for the evaluations with respect to $G S\left(\mathrm{~F}_{(1,6)}=10.752 ; \mathrm{p}=0.017\right)$ and the PDQ-39 $\left(\mathrm{F}_{(1,6)}=29.37 ; \mathrm{p}=0.002\right)$. There was no main effect for the other variables, and there was no interaction between groups and evaluation.

Considering the effect size $d$, as shown in Table 2, there is a clinically difference of large effect, comparing the initial and final period intragroup, on the SL in the CG, SLV in the GPBWS and GAS, SW in the three groups, SWV in the GPBWS, GS in the CG, and GAS and PDQ39 in the three groups. There is a medium effect for SL in the GAS and GS in the GPBWS and low effect for SL in the GPBWS and SLV in the CG. There was increased SW in the GPBWS $(p=$ $0.006)$, increased GS in the GAS ( $p=0.048)$, and decreased PDQ-39 in the CG $(\mathrm{p}=0.005)$ and GAS $(\mathrm{p}=0.006)$.

When comparing the groups independently of evaluation, there was a significant difference in SWV between the GPBWS and GAS $(p=0.030)$. In the analysis the evaluations independently of groups, the difference was for GS $(\mathrm{p}=0.048)$ and PDQ-39 $(\mathrm{p}=0.002)$, as shown in Table 3.

\section{DISCUSSION}

This clinical trial included six weeks of individual training on a treadmill, which aimed to analyse the effects of gait training on a treadmill in PD patients with PBWS and gait training with AS on the kinematic variables of gait SL, SLV, SW, SWV, and GS and on QOL. Statistical difference was showed between the initial and final periods for increased SW in the GPBWS, increase in GS in the GAS and improvement of QOL for the CG and GAS. Our hypothesis that treadmill training associated with AS or PBWS promotes significant changes in biomechanical and kinematic variables of gait was partially confirmed, since the increase in GS was only significant in GAS, and QOL improved significantly in CG as the GAS. The significant difference in SW in the GPBWS was contrary to our hypothesis.

Analysis of the magnitude of the clinical effect is a growing trend, becoming more widespread in the last quarter century. It is an alternative method for inferential statistics and is increasingly accepted by researchers from various fields, including the social sciences and medicine (Conboy, 2003). In our study, we observed a large clinical effect in each group for four of the six variables, comparing the initial and final periods (CG: SL, SW, GS, and QOL; GPBWS: SLV, SW, SWV, and QOL; GAS: SLV, SW, GS, and QOL). This clinical effect of kinematic variables after training on a treadmill may be reflected in the ability to continue walking independently. With the aging process, some strategies are adopted to maintain balance during gait, such as reducing the SL and GS. Moreover, these changes are related to a decrease in muscle strength inherent to age (Hahn, Lee, \& Chou, 2005). However, SL or GS is increased in an attempt to adapt the extent of steps appropriately to the individual. Normalizing them (Dias, Fraga, Cacho, \& Oberg, 2005), may take the elderly or patients with neurological disorders such as PD, to perform the gait more efficiently and safely. Thus, it is advantageous for people with PD to improve the variables, since the trend of the evolution of the disease changes these characteristics and is associated with reduced range of motion of joints, especially the hip, knee, and ankle, causing a slow and shuffling gait (Matsumoto et al., 2014).

Measures of variability of kinematic parameters of gait, which are usually a variation around the mean of the evaluated parameters, have been studied in the literature by means of kinematic variables, such as SL and SW. They have shown promise in predicting the risk of falls in the elderly because they are considered sensory motor deficit indicators (Callisaya, Blizzard, Mcginley, \& Srikanth, 2012). The decrease in GS has been interpreted as a compensatory mechanism for maintaining balance (Stolze et al., 2005). Three years after diagnosis, $85 \%$ of individuals with PD will probably develop walking problems and an increased risk of falling. 
Treatments have been directed to help patients retain the ability to walk independently (Matsumoto et al., 2014).

Although statistically significant differences were not found for the kinematic variables of gait in the CG, perhaps because of the small sample size, the analysis of the magnitude of the effect showed a large clinical effect by increasing the SL, GS and decreased SW in the CG. This clinical effect may have occurred because the treadmill mat stimuli facilitate movement and the start and continuation of gait, increasing the size of the steps (Nieuwboer et al., 2001). The literature suggests that this type of training stands out for its specificity for the improvement of gait in PD (Herman, Giladi, \& Hausdorff, 2009) by constant speed, regular sensory stimulation, visual feedback, and activation of the neuronal circuit which generates the central pattern of gait, which operate independently of the motor cortex and can produce rhythmic movements and motor learning (Bello, \& Fernandez-Del-Olmo, 2012).

In addition to presenting a major clinical effect on the kinematic variables of gait, the CG showed a statistically significant improvement in QOL. This corroborates the findings of Herman, Giladi, Gruendlinger, and Hausdorff (2007) which evaluated the effects of six weeks of gait training on a treadmill in the rhythmicity, functional mobility, and QOL of PD patients. They suggest that treadmill training can be used to minimize deficiencies in gait and increase the QOL of these patients. Since PD is progressive and causes movement disorders (Rodriguez et al., 2013). The loss of confidence to walk due to changes in gait and the fear of falling means that there is decreased mobility. This causes a vicious circle, because activity restriction causes decreased muscle strength and weakness of the lower limbs, leading to dependency and social isolation (Piovesan, Pivetta, \& Peixoto, 2011), which worsen QOL. Therefore, improving the general characteristics of locomotion or preventing them from getting worse can help the individual get along better without new occurrences. In this way, treadmill training helped the participants to improve their QOL.

In this study, the treadmill training associated with PBWS showed statistical significance in increasing SW and presented a large clinical effect on improvement in variable SLV (decrease) and a medium clinical effect on GS. Although the treadmill itself improves ambulation, as pointed out in the literature (Herman et al., 2009), when associated with PBWS, movement of the lower limbs is facilitated (Ganesan et al., 2014). Thus, in terms of lower limb-supported propulsion, the PBWS decreases the intensity required for projection, and it is possible that the treadmill belt passively moves the lower limb without changing muscle activation (Harris-Love et al., 2004). With respect to SW, our study found a significant increase in the GPBWS, contrary to our hypothesis, whereas the other groups experienced a large clinical effect on the decrease of SW. According to Galna, Murphy, and Morris (2010), who investigated adaptations used by people with PD while crossing an obstacle, increasing the SW is a possible strategy to overcome postural instability while crossing an obstacle. The increase in SW in this study, accompanied by a large clinical effect of increased SWV, may be related to the fact that the vest used by participants provided them a greater sense of security, especially in the GPBWS, who wore the vest fairer than the other groups. The GPBWS when evaluated in the floor, did not use the vest with the support, which may have caused greater feelings of insecurity and consequently a greater instability, evidenced by the increase in SWV. As high variability of the values in walking are related to instability in gait parameters, these values are considered indicative of instability in motor control and disorders, which are deficits of the central and peripheral nervous system (Callisaya, Blizzard, Schmidt, Mcginley, \& Srikanth, 2010).

In the analysis of the GAS gait variables, there was a significant increase in GS and a large clinical effect decreasing the SLV and SW, and medium effect to SL. Although some studies showed the AS can reduce the amount of time required for the muscles to respond to a motor control and improve the performance of the movement by increasing the excitability of spinal motor neurons (Del Olmo \& Cudeiro, 2005; Suteerawattananon et al., 2004), in this study AS associated with walking on the treadmill was not 
excelled compared to CG in clinical effect, suggesting that the proprioceptive feedback generated by the mat is sufficient to promote clinical improvement in GS variables, SL, and SW, even though the GAS was statistically highlighted with respect to GS. In a study by Baker, Rochester, and Nieuwboer (2007), the association of AS, generated by a metronome, with guidance to make great strides was effective in improving the GS and the amplitude of the pitch during the tasks. Therefore, we suggest that AS is more efficient with the gait on the ground, as in the study of Baker et al. (2007).

In the present study, comparing the GAS, that would be the AS associated with treadmill training, with the CG, the GAS had a large clinical effect on SLV, SW, and GS, whereas the GC showed the same effect for the variables SL, SW, and GS. The fact that the CG promoted virtually the same clinical effects as the GAS suggests that it is not necessary to increase the number of stimuli when using the treadmill.

Regarding the analysis of the variables of the independent walking group, we observed a significant improvement in GS and QOL. When GS is considered slow, it is predictive of early death, disability, falls, and hospitalization/institutionalization in older people living in the community (Pamoukdjian et al., 2015). The reduction is due to the slowness of the gravity centre displacement, decreasing of the vertical component of the ground reaction force (Liu et al., 2006) and by the large lateral displacement of the pressure centre in the gait start (Gantchev, Viallet, Aurenty, \& Massion, 1996). The decline of the GS is also due to decreased elasticity of the muscles and reduced joint mobility and muscle strength (Pijnappels, Bobbert, \& Van Dieën, 2001), suggesting that the proposed training improved these components, aiding in the improvement of GS. In addition, improving the GS can help people with PD in their daily activities, such as getting to the phone in time or reaching the bathroom in the case of an emergency, which improves QOL.

In this study, the final averages of PDQ-39 were lower than the initial questionnaire, being significant for the CG and GAS and a large clinical effect was showed for the three groups studied.
QOL, assessed by the PDQ-39, can be considered a multidimensional concept that reflects a subjective evaluation of patients' personal satisfaction with their life (Camargos, Cópio, Sousa, \& Goulart, 2004). In PD, when there is deterioration of this concept, motors aspects can be considered largely responsible, since they act as precursors of limitations in other dimensions (Camargos et al., 2004). Therefore, the goal of improving motor function in PD patients is important to change their perceived QOL (Lana et al., 2007).

The limitations of this study were related to the small number of participants in each group and the absence of monitoring of the kinematic variables of gait during training on a treadmill, to show if there was some improvement during training so that it could be transferred to the gait on the floor. Another limitation was in relation to the lack of evaluation, mobility testing or followup of more specific daily activities, to analyse the effect of training also in these variables. For future studies, we suggest re-evaluating the variables after a training period for completing the analysis of the maintenance of gains.

\section{CONCLUSION}

We conclude that, among the studied conditions, there was a significant improvement for the groups: AS in GS and QOL, CG in QOL and GPBWS did not improve. Thus, considering the clinical effect, treadmill training, independent of the stimuli, is clinically advantageous for gait improvements and QOL.

\footnotetext{
Acknowledgments:

Coordenação de Aperfeiçoamento de Pessoal de Nível Superior - Capes
}

\begin{tabular}{l}
\hline Conflict of interests: \\
Nothing to declare. \\
\hline
\end{tabular}

\begin{tabular}{l}
\hline Funding: \\
Nothing to declare. \\
\hline
\end{tabular}

\section{REFERENCES}

Abbruzzese, G., Marchese, R., Avanzino, L., \& Pelosin, E. (2016). Rehabilitation for Parkinson's disease: Current outlook and future 
challenges. Parkinsonism Related Disorders, 22 Suppl 1, S60-64. doi:10.1016/j.parkreldis.2015.09.005

Baker, K., Rochester, L., Nieuwboer, L., \& Nieuwboer, A. (2007). The immediate effect of attentional, auditory, and a combined cue strategy on gait during single and dual tasks in parkinson's disease. Archives Physical Medicine and Reabilitation, 88(12), 1593-1600.

Bello, O., \& Fernandez-Del-Olmo, M. (2012). How does the treadmill affect gait in Parkinson's disease? Current Aging Science, 5(1), 28-34.

Bello, O., Sanchez, J. A., \& Fernandez-del-Olmo, M. (2008). Treadmill walking in Parkinson's disease patients: adaptation and generalization effect. Movement Disorders, 23(9), 1243-1249. doi:10.1002/mds.22069

Bello, O., Sanchesz, J.A., Lopez-Alonso, V., Márquez, G., Morenilla, L., Castro, X., Giraldez, M., SantosGarcía, D., \& Fernandez-del-Olmo M. (2013). The effects of treadmill or overground walking training program on gait in Parkinson's disease. Gait Posture, 38(4), 590-595.

Brucki, S. M. D., Nitrini, R., Caramelli, P., Bertolucci, P. H. F., \& Okamoto, I. H. (2003). Sugestões para o uso do mini-exame do estado mental no Brasil. Arquivos de Neuro-Psiquiatria, 61 (3B), 777-781.

Callisaya, M. L., Blizzard, L., McGinley, J. L., \& Srikanth, V. K. (2012). Risk of falls in older people during fast-walking--the TASCOG study. Gait Posture, 36(3), 510-515. doi:10.1016/j.gaitpost.2012.05.003

Callisaya, M. L., Blizzard, L., Schmidt, M. D., McGinley, J. L., \& Srikanth, V. K. (2010). Ageing and gait variability--a population-based study of older people. Age and Ageing, 39(2), 191-197. doi:10.1093/ageing/afp250

Camargos, A. C. R., Cópio, F. C. Q., Sousa, T. R. R., \& Goulart, F. (2004) O impacto da Doença de Parkinson na qualidade de vida: uma revisão de literatura. Revista Brasileira de Fisioterapia, 8(3), 267-272

Carod-Artal, F. J., Martinez-Martin, P., \& Vargas, A. P. (2007). Independent validation of SCOPApsychosocial and metric properties of the PDQ39 Brazilian version. Movement Disorders, 22(1), 91-98. doi:10.1002/mds. 21216

Cohen, J. (1992). A power primer. Psychological bulletin $112,155-159$.

Conboy, J. E. (2003). Algumas medidas típicas univariadas da magnitude do efeito. Análise Psicológica, 2(21), 145-158

Del Olmo, M. F., \& Cudeiro, J. (2005). Temporal variability of gait in Parkinson disease: effects of a rehabilitation programme based on rhythmic sound cues. Parkinsonism \& Related Disorders, 11(1), 25-33.

Dingwell, J. B., \& Marin, L. C. (2006). Kinematic variability and local dynamic stability of upper body motions when walking at different speeds. Journal of Biomechanics, 39(3), 444-452.
Dias, N. P., Fraga, D. A., Cacho, E. W. A., \& Oberg, T. D. (2005). Treino de marcha com pistas visuais no paciente com Doença de Parkinson. Fisioterapia em Movimento, 18(4), 43-51.

Earhart, G. M., \& Williams, A. J. (2012). Treadmill training for individuals with Parkinson disease. Physical Therapy, 92(7), 893-897. doi:10.2522/ptj.20110471

Galna, B., Murphy, A. T., \& Morris, M. E. (2010). Obstacle crossing in people with Parkinson's disease: foot clearance and spatiotemporal deficits. Human Movement Science, 29(5), 843-852. doi:10.1016/j.humov.2009.09.006

Ganesan, M., Sathyaprabha, T. N., Gupta, A., \& Pal, P. K. (2014). Effect of partial weight-supported treadmill gait training on balance in patients with Parkinson disease. Physical Medicine and Rehabilitation, 6(1), 22-33. doi:10.1016/j.pmrj.2013.08.604

Gantchev, N., Viallet, F., Aurenty, R., \& Massion, J. (1996). Impairment of posturo-kinetic coordination during initiation of forward oriented stepping movements in parkinsonian patients. Electroencephalography and Clinical Neurophysiology, $101(2), 110-120$.

Hahn, M. E., Lee, H. J., \& Chou, L. S. (2005). Increased muscular challenge in older adults during obstructed gait. Gait \& Posture, 22(4), 356-361. doi:10.1016/j.gaitpost.2004.11.012

Harris-Love, M. L., Macko, R. F., Whitall, J., \& Forrester, L. W. (2004). Improved hemiparetic muscle activation in treadmill versus overground walking. Neurorehabilitation and Neural Repair, 18(3), 154-160. doi: $10.1177 / 0888439004267678$

Herman, T., Giladi, N., Gruendlinger, L., \& Hausdorff, J. M. (2007). Six weeks of intensive treadmill training improves gait and quality of life in patients with Parkinson's disease: a pilot study. Archives of Physical Medicine and Rehabilitation, 88(9), 1154-1158. doi:10.1016/j.apmr.2007.05.015

Herman, T., Giladi, N., \& Hausdorff, J. M. (2009). Treadmill training for the treatment of gait disturbances in people with Parkinson's disease: a mini-review. Journal of neural transmission (Vienna), 116(3), 307-318. doi:10.1007/s00702008-0139-z

Hoehn, M. M., \& Yahr, M. D. (1967). Parkinsonism: onset, progression and mortality. Neurology, 17(5), 427-442.

Lana, R.C., Álvares, L. M. R. S., Nasciutti-Prudente, C., Goulart, F. R. P., Teixeira-Salmela, L. F., \& Cardoso, F. E. (2007). Percepção da qualidade de vida de indivíduos com Doença de Parkinson através do PDQ-39. Revista Brasileira de Fisioterapia, 11 (5), 397-402.

Liu, W., McIntire, K., Kim, S. H., Zhang, J., Dascalos, S., Lyons, K. E., \& Pahwa, R. (2006). Bilateral subthalamic stimulation improves gait initiation in patients with Parkinson's disease. Gait $\mathcal{E}$ 
Posture, $\quad 23(4), \quad 492-498$. doi:10.1016/j.gaitpost.2005.06.012

Matsumoto, L., Magalhães, G., Antunes, G. L., \& Torriani-Pasin, C. (2014). Efeitos do estímulo acústico rítmico na marcha de pacientes com Doença de Parkinson. Revista Neurociências, 22 (3), 404-409.

Miller, R. A., Thaut, M. H., McIntosh, G. C., \& Rice, R. R. (1996). Components of EMG symmetry and variability in parkinsonian and healthy elderly gait. Electroencephalography and Clinical Neurophysiology, 101 (1), 1-7.

Mirelman, A., Rochester, L., Reelick, M., Nieuwhof, F., Pelosin, E., Abbruzzese, G., ... Hausdorff, J. M. (2013). V-TIME: a treadmill training program augmented by virtual reality to decrease fall risk in older adults: study design of a randomized controlled trial. BMC Neurology, 13, 15. https://doi.org/10.1186/1471-2377-13-15

Miyai, I., Fujimoto, Y., Yamamoto, H., Ueda, Y., Saito, T., Nozaki, S., \& Kang, J. (2002). Long-term effect of body weight-supported treadmill training in Parkinson's disease: a randomized controlled trial. Archives of Physical Medicine and Rehabilitation, 83(10), 1370-1373.

Nadeau, A., Pourcher, E., \& Corbeil, P. (2014). Effects of $24 \mathrm{wk}$ of treadmill training on gait performance in Parkinson's disease. Medicine and Science in Sports and Exercise, 46(4), 645-655. doi:10.1249/MSS.0000000000000144

Nieuwboer, A., De Weerdt, W., Dom, R., Truyen, M., Janssens, L., \& Kamsma, Y. (2001). The effect of a home physiotherapy program for persons with Parkinson's disease. Journal of Rehabilitation Medicine, 33(6), 266-272.

Novaes, R. D., Miranda, A. S., \& Dourado, V. Z. (2011). Velocidade usual da marcha em brasileiros de meia idade e idosos. Revista Brasileira de Fisioterapia, 15(2), 117-122

Pamoukdjian, F., Paillaud, E., Zelek, L., Laurent, M., Lévy, V., Landre, T., \& Sebbane, G. (2015).
Measurement of gait speed in older adults to identify complications associated with frailty: A systematic review. Journal of Geriatric Oncology, 6(6), 484-496. doi:10.1016/j.jgo.2015.08.006

Paterson, K. L., Lythgo, N. D., \& Hill, K. D. (2009). Gait variability in younger and older adult women is altered by overground walking protocol. Age and Ageing, 38(6), 745-748. doi:10.1093/ageing/afp159

Pijnappels, M., Bobbert, M. F., \& van Dieën, J. H. (2001). Changes in walking pattern caused by the possibility of a tripping reaction. Gait $\mathcal{E}$ Posture, 14(1), 11-18.

Piovesan, A. C., Pivetta, H. M. F., \& Peixoto, J. M. B. (2011). Fatores que predispõem a quedas em idosos residentes na região oeste de Santa Maria, RS. Revista Brasileira de Geriatria e Gerontologia, 14(1), 75-83.

Rodriguez, K. L., Roemmich, R. T., Cam, B., Fregly, B. J., \& Hass, C. J. (2013). Persons with Parkinson's disease exhibit decreased neuromuscular complexity during gait. Clinical Neurophysiology, 124(7), 1390-1397. doi:10.1016/j.clinph.2013.02.006

Scalzo, P. L., Flores, C. R., Marques, J. R., Robini, S. C., \& Teixeira, A. L. (2012). Impact of changes in balance and walking capacity on the quality of life in patients with Parkinson's disease. Arquivos de Neuro-Psiquiatria, 70(2), 119-124.

Stolze, H., Klebe, S., Baecker, C., Zechlin, C., Friege, L., Pohle, S., \& Deuschl, G. (2005). Prevalence of gait disorders in hospitalized neurological patients. Movement Disorders, 20(1), 89-94. doi: $10.1002 / \mathrm{mds} .20266$

Suteerawattananon, M., Morris, G. S., Etnyre, B. R., Jankovic, J., \& Protas, E. J. (2004). Effects of visual and auditory cues on gait in individuals with Parkinson's disease. Journal of the Neurological Sciences, $\quad$ 219(1-2), 63-69. doi:10.1016/j.jns.2003.12.007 